\title{
Quorum sensing inhibitory activity of sub-inhibitory concentrations of $\beta$-lactams
}

\author{
Somaia A. El-Mowafy ${ }^{1}$, Khaled H. Abd El Galil', El-Sayed E. Habib ${ }^{3}$, Mona I. Shaaban ${ }^{3}$
}

1. Microbiology Department, Faculty of Pharmacy, Mansoura University, Mansoura, 35516 Egypt.

2. Pharmaceutical Sciences Department, Faculty of Pharmacy, Beirut Arab University, Beirut, Lebanon. Department of Microbiology and Immunology, Faculty of Pharmacy, Mansoura University, Mansoura, 35516 Egypt.

3. Department of Pharmaceutics and Pharmaceutical Biotechnology, Faculty of Pharmacy, Taibah University, Al Madinah Al Munawwarah 30078, Saudi Arabia. Department of Microbiology and Immunology, Faculty of Pharmacy, Mansoura University, Mansoura, 35516 Egypt.

\begin{abstract}
Introduction: The virulence factors of Psendomonas aeruginosa are under the control of quorum sensing (QS) signals. Hence, interference with QS prevents its pathogenesis.

Objective: The aim of the present research is to assess the influence of some $\beta$-lactam antibiotics on cell communication and the release of different virulence factors.

Methods: The minimal inhibitory concentrations of ceftazidime, cefepime and imipenem were evaluated by microbroth dilution method. The effect of sub-inhibitory concentration of the tested antibiotics on QS signals was investigated using reporter strain assay. In addition, different virulence factors (elastase, protease, pyocyanin and hemolysin) were estimated in the presence of their sub-inhibitory concentrations.

Results: Low concentrations of ceftazidime, cefepime and imipenem caused significant elimination of the QS signals $3 \mathrm{OH}-$ C12-HSL and C4-HSL up to 1/20 MIC. Furthermore, low concentrations of the tested antimicrobials suppressed virulence factors elastase and hemolysin. Moreover, 1/20 of their MICs reduced elastase, protease, pyocyanin and hemolysin.

Conclusion: Utilization of $\beta$-lactam antibiotics at low concentrations could be an effective approach for prevention and treatment of $P$. aeruginosa infection.
\end{abstract}

Keywords: Quorum sensing inhibition, $\beta$-lactams, Pseudomonas aeruginosa

DOI: https://dx.doi.org/10.4314/ahs.v17i1.25

Cite as: El-Mowafy SA, Abd El Galil KH, Habib E-SE, Shaaban MI. Quorum sensing inbibitory activity of sub-inbibitory concentrations of B-lactams. Afri Health Sci. 2017;17(1): 199-207. bttps:// dx.doi.org/10.4314/ahs.v17i1.25

\section{Introduction}

Pseudomonas aeruginosa is an opportunistic human pathogen with remarkable metabolic versatility. Infections with $P$. aeruginosa are common in compromised patients suffering from cystic fibrosis, severe burns, deep wounds, in addition to patients having urinary tract infections. $P$. aeruginosa produces various virulence attributes, including
Corresponding author:
Mona I. Shaaban,
Department of Pharmaceutics
and Pharmaceutical
Biotechnology, Faculty of Pharmacy,
Taibah University,
Al Madinah Al Munawwarah 30078, Saudi Arabia
Phone: +201092535893; fax: (+2) 050-2246253
E-mail: mona_ibrahem@mans.edu.eg,
sarafawzy2002@yahoo.com

biofilm, toxins and enzymes such as pyocyanin, protease, elastase, and rhamnolipids ${ }^{1}$.

$P$. aeruginosa exhibits its virulence behavior via quorum sensing $(\mathrm{QS})^{2}$. The common $\mathrm{QS}$ systems in P. aeruginosahave been assigned las and rhl. QS circuits in $P$. aeruginosa are connected by signaling molecules called autoinducers. The las system is composed of the synthase gene lasI and its transcriptional regulatory protein LasR. Its autoinducer is called $\mathrm{N}$-(3-oxododecanoyl) homoserine lactone $(3 \mathrm{OH}-\mathrm{C} 12-\mathrm{HSL})$. Similarly, the rhl system consists of rhlI synthase, and its transcriptional regulatory protein LasR. Also, it possesses autoinducer molecule N-butyryl homoserine lactone (C4-HSL) ${ }^{2,3}$. When bacterial growth reaches a specific threshold, the signals acyl homoserine lactones (AHL) are released and stimulates the expression of virulence genes ${ }^{4}$. Both the las and rhl systems are coregulated, and las system is dominant over the rhl pathway. Hence, inhibition of these signaling molecules hinders the pathogenicity of $P$. aeruginosa, and could be used for prevention or treatment of its infections ${ }^{5}$. 
Several plants have QS inhibiting activities. Medicinal plants $^{6}$ and edible plants ${ }^{7}$ exhibited QSI effects. Also, Streptomyces coelicoflavus isolated from soil microbiota produced 1 H-pyrrole-2-carboxylic acid with QSI effect ${ }^{8}$. Synthetic molecules and peptides exhibited QSI activity ${ }^{9}$. Previous studies focused on the effect of some antimicrobials such as aminoglycosides and quinolones on QS of $P$. aeruginosa. Azithromycin showed significant elimination of QS and virulence factors of $P$. aeruginosa ${ }^{10}$. The present study focused on the effect of sub-inhibitory concentrations of some $\beta$-lactam antibiotics on QS of $P$. aeruginosa. Furthermore, virulence factors of $P$. aeruginosa were assessed in the presence of sub-inhibitory concentrations of the tested antibiotics.

\section{Materials and methods}

\section{Bacterial strains, growth media and conditions}

The wild strain $P$. aeruginosa PAO1 was used for the assay of QSI effects of the tested antibiotics. Two reporter strains; P. aeruginosa pME3846 (rhlI-lacZ; Tc ${ }^{\mathrm{r}}$ ) and E. coli MG4/pKDT17 (lasB::lacZplac-lasR Ap ${ }^{\mathrm{r}}$ )2,3 were used for the assessment of rhlI and lasI/R, respectively in the presence and absence of the tested antibacterials. The QS deficient $P$. aeruginosa isolate PAO-JP2 double mutant ( $\Delta$ lasI::Tn10,Tcr; $\Delta$ rhlI::Tn5012, Hgr) was included as a negative control ${ }^{11}$. All bacterial cultures were grown in Luria Bertani medium (LB broth; tryptone 1\%, yeast extract $0.5 \%$, and $\mathrm{NaCI} 1 \%$ ) at $37^{\circ} \mathrm{C}$.

\section{Determination of minimal inhibitory concentration}

Minimal inhibitory concentrations (MICs) of the studied $\beta$-lactams: cefepime (Cfp), ceftazidime (Cft), and imipenem (Imp), were estimated by broth microdilution method (CLSI, 2014). Two-fold serial dilutions of each antibiotic were prepared and inoculated with $0.1 \mathrm{ml}$ of PAO1 inoculum contained $5 \times 10^{6} \mathrm{CFU} / \mathrm{ml}$ and incubated at $37^{\circ} \mathrm{C}$ for $24 \mathrm{~h}$. Values of MIC were recorded as the lowest concentration of the antibiotic at which there was no visible growth of the organism ${ }^{12}$.

\section{Determination of the viable count of $P$. aeruginosa- PAO1}

The viability of $P$. aeruginosa PAO1 wild type was examined in the presence of sub-inhibitory concentrations $(1 / 4$ MIC) of the tested $\beta$-lactams using pour plate counting method and cell proliferation was checked before supernatant collection ${ }^{13}$. Similarly, the viable count of the untreated cells was performed and compared to the treated cultures.

\section{Preparation of the supernatant}

$P$. aeruginosa $\mathrm{PAO} 1$ was propagated in LB broth containing $1 / 4,1 / 8$ and $1 / 20 \mathrm{MIC}$ of each antibiotic. P. aeruginosa PAO1 was also grown without antimicrobial agents as the positive control and PAO-JP2 was propagated under the same conditions as the negative control ${ }^{14}$. The supernatants of the treated and untreated Pseudomonas cultures were separated by centrifugation at $8.000 \mathrm{rpm}$ for $10 \mathrm{~min}$ at $4^{\circ} \mathrm{C}$. Cell-free supernatants were then stored at $-20^{\circ} \mathrm{C}$ to be used for estimation of AHLs and assay of various virulence factors ${ }^{15}$.

\section{Effect of $\beta$-lactams on QS signal molecules}

The QS signals 3OH-C12-HSL and C4-HSL were detected in treated and untreated cultures of PAO1, respectively. The overnight growth of the reporter strains E. coli MG4 (pKDT17) and P. aeruginosa (pME3846) were diluted up to OD600 of 0.1. The previously prepared cell-free supernatant $(1 \mathrm{ml})$ was mixed with $0.5 \mathrm{ml}$ of E. coli MG4 and $1 \mathrm{ml} P$. aeruginosa $\mathrm{pME} 3846$. Cells were propagated till they reached $0.3-0.4$ at OD600 then pelleted. $\beta$-galactosidase was estimated according to Miller assay meth$\operatorname{od}^{16}$.

\section{Effect on virulence factors}

The effect of the tested $\beta$-lactams on the virulence determinants of $P$. aeruginosa PAO1 was estimated using the prepared supernatants both in the presence and absence of tested $\beta$-lactams.

\section{Evaluation of LasB elastase}

The supernatant of $P$. aeruginosa was mixed with $10 \mathrm{mg}$ of elastin Congo red (ECR, Sigma Chemicals, St. Louis, USA) in ECR buffer (100 mM Tris buffer, $\mathrm{pH}$ 7.2). The insoluble substrate was centrifuged and the absorbance of the supernatant was estimated at $495 \mathrm{~nm}^{17}$.

\section{Total protease production}

Total proteolytic effects of the treated and untreated cells were detected using modified skim milk method ${ }^{18}$. Cultures supernatants of PAO1 were mixed with skim milk $(1.25 \%)$ at $1: 2$ ratio and reaction was incubated at $37^{\circ} \mathrm{C}$ for $30 \mathrm{~min}$. The turbidity of the solution was determined at OD $600 \mathrm{~nm}$. The results were calculated as relative protease production to the untreated PAO1 strain. 


\section{Determination of hemolysin production}

The hemolysin test was performed by incubating $700 \mu \mathrm{l}$ of $2 \%$ erythrocytes suspension with $0.5 \mathrm{ml}$ of PAO1 supernatant for $2 \mathrm{~h}$ at $37^{\circ} \mathrm{C}$. The suspension was centrifuged and the produced hemoglobin was estimated by measuring the OD at $540 \mathrm{~nm}$. The negative control; RBCs in LB broth was evaluated under the same conditions. The total RBCs lysed were detected using $0.1 \% \mathrm{SDS}^{19}$.

\section{Pyocyanin assay}

King A media (peptone 2\%, K2SO4 1\%, and $\mathrm{MgCl} 2$ $0.14 \%$ ) supplied with sub-MICs of $\beta$-lactams was inoculated and propagated at $37^{\circ} \mathrm{C}$ for $48 \mathrm{~h}$ in triplicates. Media without antibiotics was also inoculated with PAO1 and propagated as a positive control. The culture was centrifuged and pyocyanin was extracted using chloroform in acidic $\mathrm{pH}$ and the red layer was separated. Its absorbance was measured at $520 \mathrm{~nm}$ and pyocyanin concentration was expressed as $\mu \mathrm{g} / \mathrm{ml}$ by multiplying the OD520 by $17.072^{20}$.

\section{Statistical analysis}

The mean of three independent experiments and the standard deviation of the mean were calculated by Excel data package. $P$. aeruginosa treated with $\beta$-lactams were compared to the untreated control using GraphPad Instate software (version 3.05) and Tukey-Kramer multiple-comparison test. Significant difference between treated and untreated culture was assigned when the probability value was $\mathrm{P}<0.05$ or $\mathrm{P}<0.01$.

\section{Results}

Growth inhibitory concentrations for $P$. aeruginosa PAO1 Table (1) showed the MIC values of tested $\beta$-lactams and the mean value for their sub-inhibitory concentrations used for testing QSI effect and virulence factors.

\section{Table (1): Minimal inhibitory concentration (MIC) values of the tested $\beta$-lactam and their sub-inhibitory concentrations}

\begin{tabular}{lllll}
\hline Compounds & MIC value & $\mathbf{1 / 4}$ MIC & $\mathbf{1 / 8}$ MIC & $\mathbf{1 / 2 0}$ MIC \\
\hline Cefepime (Cfp) & $200(\mu \mathrm{g} / \mathrm{ml})$ & 50 & 25 & 10 \\
Ceftazidime (Cft) & $1.0(\mu \mathrm{g} / \mathrm{ml})$ & 0.2 & 0.1 & 0.05 \\
Imipenem(Imp) & $1.0(\mu \mathrm{g} / \mathrm{ml})$ & 0.2 & 0.1 & 0.04 \\
\hline
\end{tabular}

Effect of sub-inhibitory concentrations of the tested $\beta$-lactams on the growth of $P$. aeruginosa PAO1

Viable counts of $P$. aeruginosa PAO1 treated with sub-inhibitory concentrations of cefepime, ceftazidime, and imipenem was performed using pour plate method. The tested concentrations (1/4 MIC) did not alter bacterial growth compared to the control untreated cultures and the same bacterial count of untreated PAO1 $\left(161 \pm 21 \mathrm{X} 10^{7}\right.$ $\mathrm{CFU} / \mathrm{ml})$.

\section{Inhibition of quorum sensing signal molecules} Effect on 3OH-C12-HSL

Untreated $P$. aeruginosa $\mathrm{PAO} 1$ produced the highest $\beta$-galactosidase activity (3125 Miller units). Double mutant strain devoid of QS genes (PAO-JP2) showed the lowest level of $\beta$-galactosidase (39 Miller units). The level of $3 \mathrm{OH}-\mathrm{C} 12-\mathrm{HSL}$ was significantly reduced by all sub-MICs of cefepime (66\%-76\% inhibition) and imipenem (63\%$78 \%$ ). However, ceftazidime exhibited less significant effect ranged from $54 \%$ at $1 / 4 \mathrm{MIC}$ to $47 \%$ at $1 / 8 \mathrm{MIC}$, while ceftazidime (1/20 MIC) did not affect 3OH-C12HSL yield (Fig. 1) as compared to the untreated PAO1 strain. 


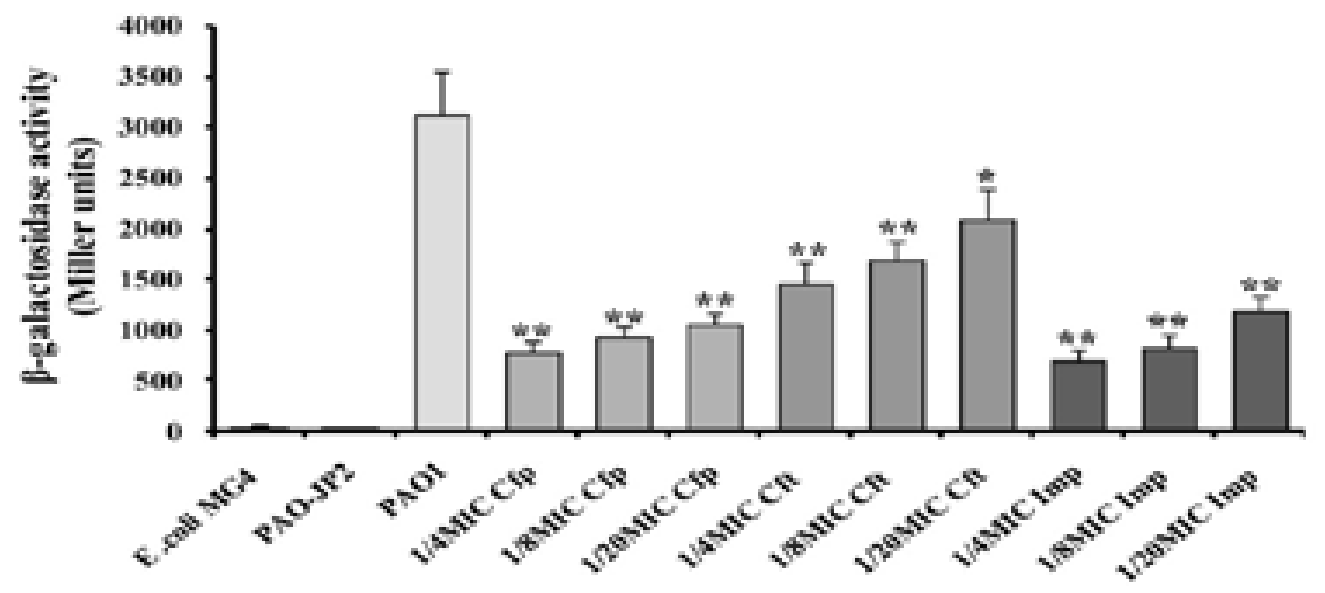

Figure (1): $\beta$-galactosidase assay of $30 \mathrm{H}-\mathrm{C}_{12}$-HSL. Effect of sub-MICs of cefepime (Cfp), ceftazidime (Cft), and imipenem (Imp) on $30 \mathrm{H}-\mathrm{C}_{12}-\mathrm{HSL}$ production (**, highly significant $P<0.01$ and *, significant $P<0.05$ ).

\section{Effect on C4-HSL}

C4-HSL level was significantly reduced $(\mathrm{P}<0.01)$ by the assayed antibiotics at their sub-inhibitory concentrations with different degrees of signals inhibition (Fig. 2) as compared to the untreated $P$. aeruginosa PAO1. C4-HSL levels were significantly lowered by sub-MICs of cefepime by $47-68 \%$ inhibition, ceftazidime by $(40 \%-52 \%)$, and imipenem by $(47-56 \%)$. Double mutant strain showed the lowest level of $\beta$-galactosidase activity (85 Miller units). All tested antibiotics produced no effect on both reporter strains.

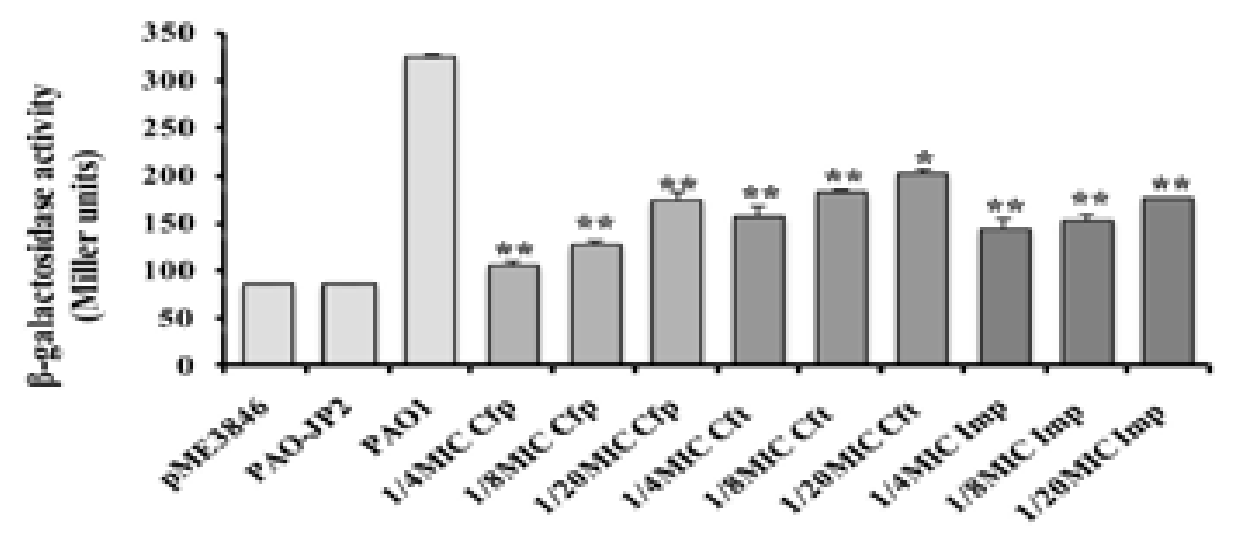

Figure (2): $\beta$-galactosidase assay of $\mathrm{C}_{4}$-HSL. Effect of sub-MICs of cefepime (Cfp), ceftazidime (Cft), and imipenem (Imp) on the release of $\mathrm{C}_{4}-\mathrm{HSL}$ (**, highly significant $P<0.01$ and $*$, significant $P<0.05$ ).

Effect of sub-inhibitory concentrations on virulence factors of $P$. aeruginos PAO1

\section{Effect on elastase enzyme}

Significant reduction in elastase production was detected when $P$. aeruginosa PAO1 was grown in presence of sub-inhibitory concentrations of cefepime $(61 \%-70 \%$ inhibition) and imipenem (52\%-66\%) with $\mathrm{P}<0.01$. However, ceftazidime exhibited significant effect ranging from $54 \%$ at $1 / 4$ MIC with $\mathrm{P}<0.01$ to $42 \%$ at $1 / 8 \mathrm{MIC}$ $(\mathrm{P}<0.05)$, while $1 / 20 \mathrm{MIC}$ ceftazidime did not affect the elastase level (Fig. 3). 


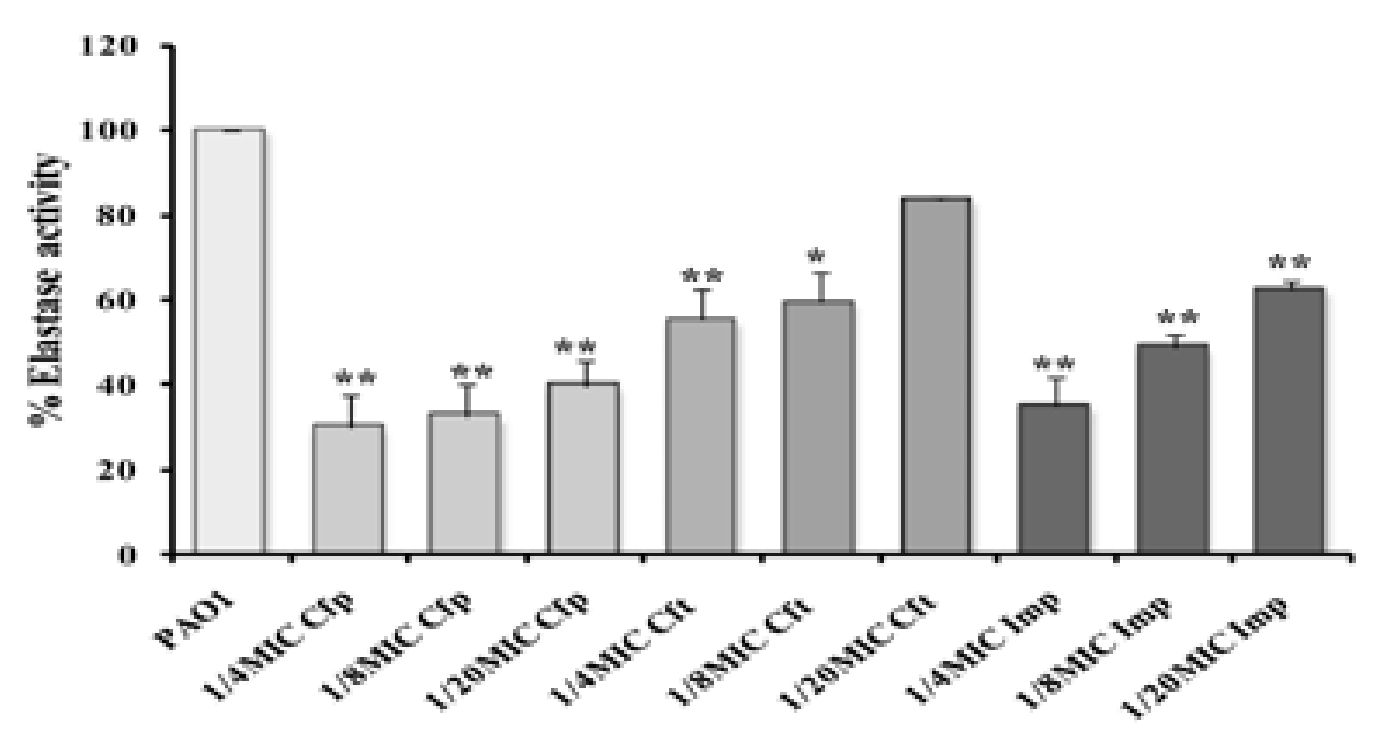

Figure (3): Relative production of elastase from P. aeruginosa PAO1 grown with sub-MICs of cefepime (Cfp), ceftazidime (Cft), and imipenem (Imp) (**, highly significant $P<0.01$ and *, significant $P<0.05)$.

\section{Effect on total protease}

There was significant lowering in total protease production in cultures supplied with sub-MICs of cefepime (51-61\% decrease) and imipenem (50-62\%) with $\mathrm{P}<0.01$.
However, ceftazidime exhibited variable reduction levels ranged from $62 \%$ to $54 \%$ at $1 / 4$ and $1 / 8$ MIC, respectively $(\mathrm{P}<0.01)$. However, it showed less effect at $1 / 20$ MIC (39\%, P<0.05) (Fig. 4).

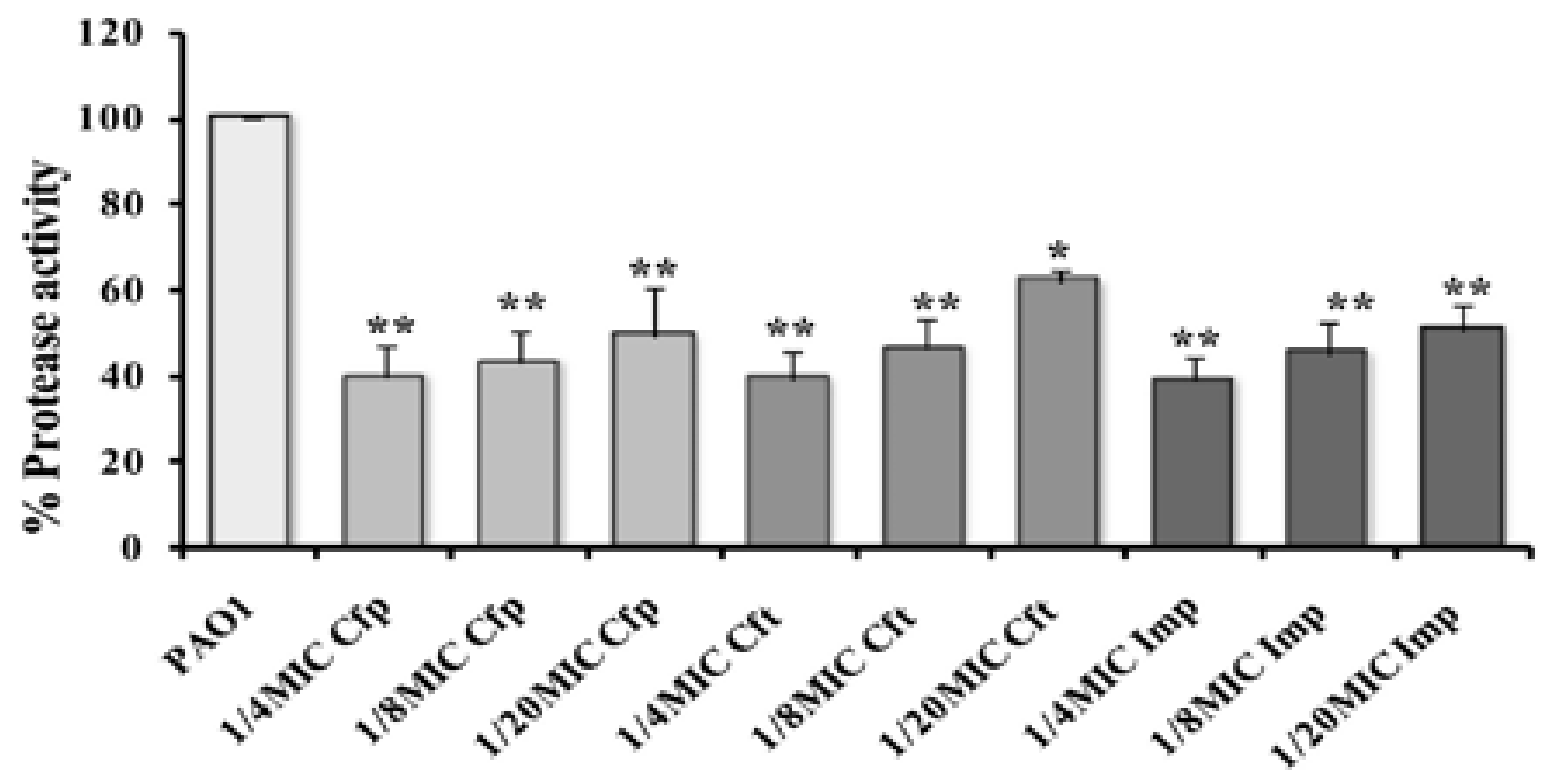

Figure (4): Effect on the production of total protease in PAO1 by sub-MICs of cefepime (Cfp), ceftazidime (Cft), and imipenem (Imp) (**, highly significant $P<0.01$ and *, significant $P<0.05)$.

\section{Effect on hemolysin production}

The effect on hemolytic activity was expressed as percentage lysis of RBCs by PAO1culture supernatants treated with sub-inhibitory concentrations, as compared to lysis obtained by the untreated PAO1 supernatant ${ }^{19}$. Significant reduction in hemolysin production $(\mathrm{P}<0.01)$ was recorded with sub-MICs of cefepime (69-83\%), ceftazidime (58-72\%), and imipenem (55-69\%) (Fig. 5). 


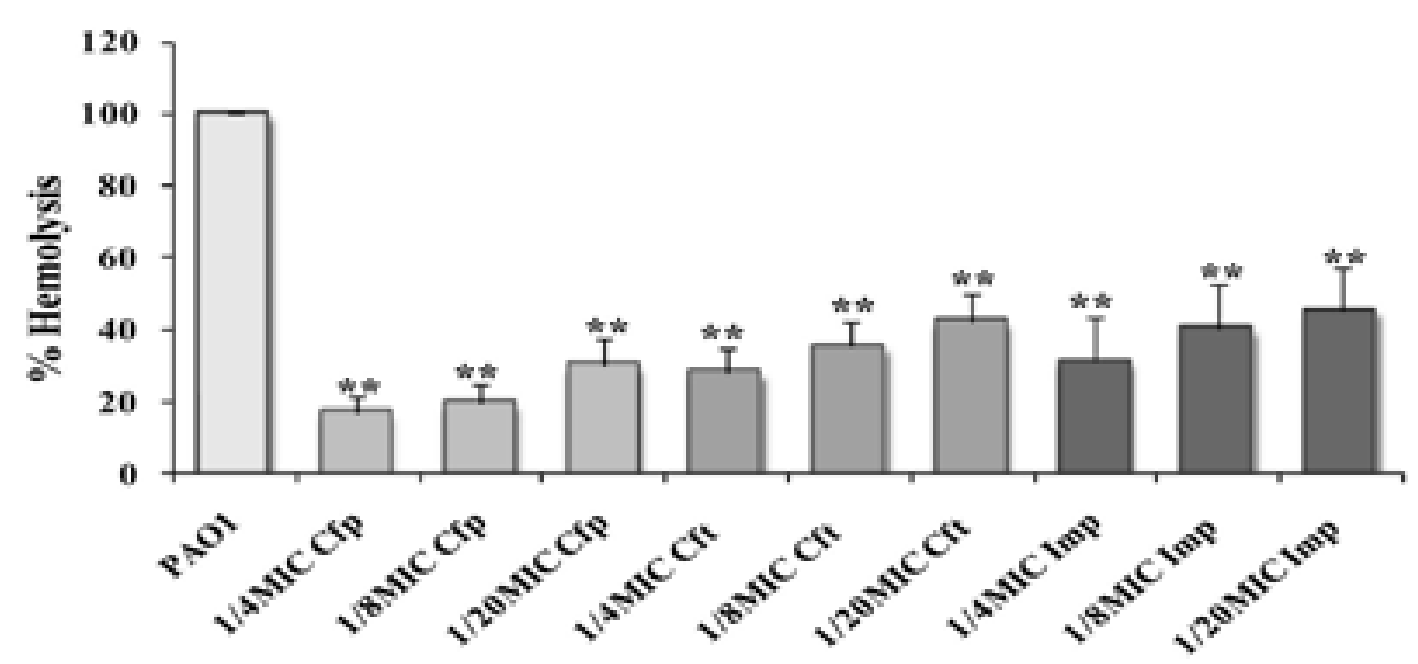

Figure (5): Inhibition of hemolytic activity in P. aeruginosa PAO1 using sub-inhibitory concentrations of cefepime (Cfp), ceftazidime (Cft), and imipenem (Imp) (**, highly significant $P<0.01$ and *, significant $\boldsymbol{P}<0.05)$.

\section{Elimination of pyocyanin production}

The effect of the tested antibiotics on the production of pyocyanin pigment from PAO1 cells was assessed. Pyocyanin production was significantly reduced by sub-MICs of cefepime (63-73\%), 1/4 and 1/8 MIC of imipenem

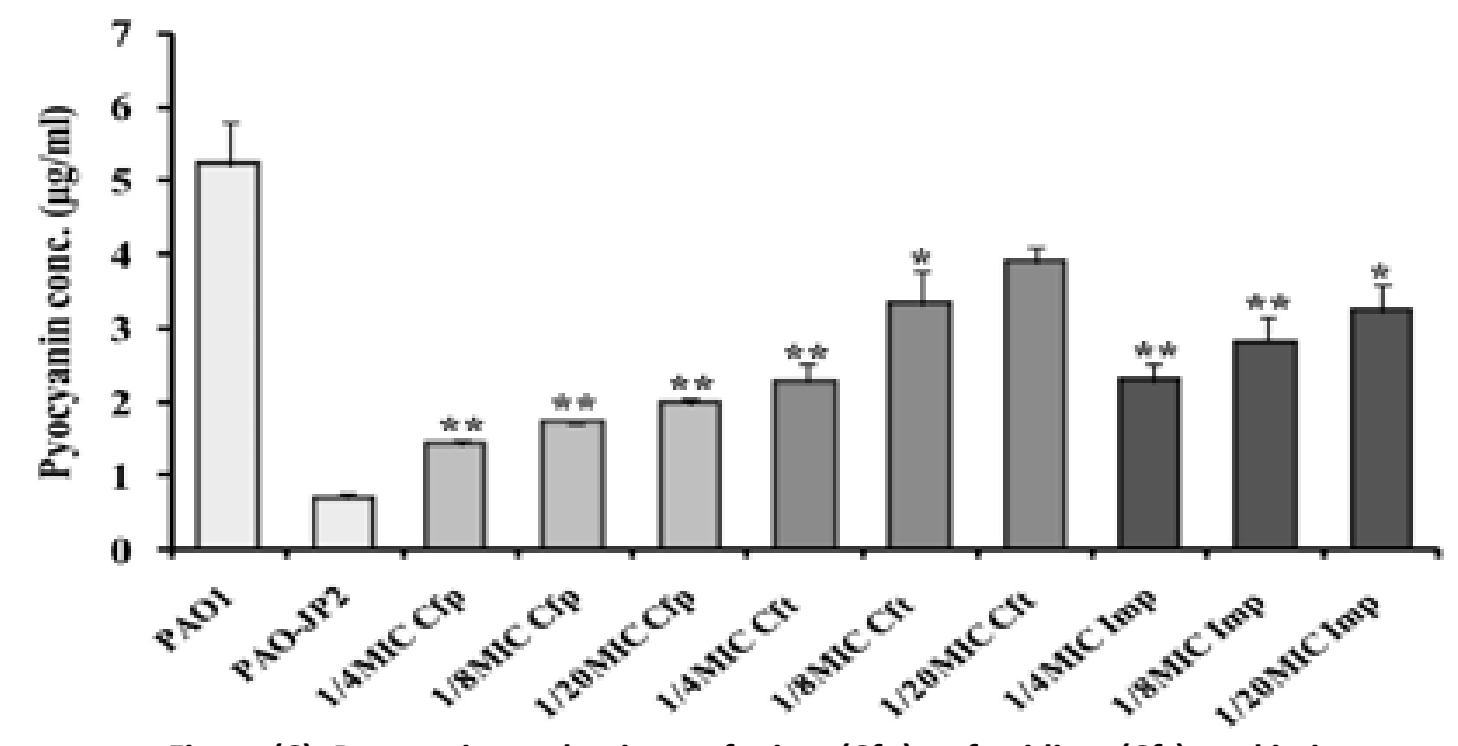

Figure (6): Pyocyanin production; cefepime (Cfp), ceftazidime (Cft), and imipenem (Imp) compared to untreated standard strain PAO1. (**, highly significant $P<0.01$ and *, significant $P<0.05$ ).

\section{Discussion}

P. aeruginosa is one of the main causes of the persistent urinary tract, respiratory tract, burn and wound infections $^{21}$. Pathogenesis of $P$. aeruginosa is attributed to secreted virulence factors such as elastase, proteases, and pyocyanin. Its pathogenesis is also disseminated through bacterial adhesion and biofilm formation ${ }^{5}$. QS is the key
(51-57\%) with $\mathrm{P}<0.01$, and $1 / 20$ MIC of imipenem by $40 \%(\mathrm{P}<0.05)$. However, ceftazidime exhibited significant reduction ranged from $57 \%$ at $1 / 4$ MIC to $37 \%$ at 1/8 MIC, while 1/20 MIC did not affect pyocyanin yield (Fig. 6). On the other hand, PAO-JP2 showed the lowest pyocyanin level $(0.6 \mu \mathrm{g} / \mathrm{ml})$

regulator for Pseudomonas virulence and biofilm formation. Hence, reduction in bacterial communication by QS inhibitors results in reduced levels of virulence factors ${ }^{22}$. Therefore, targeting QS cascade developed a new therapeutic approach for control of Psendomonas infections. This study showed that the sub-inhibitory concentrations of the three tested $\beta$-lactams exhibited significant 
elimination of QS signals. Quantitative assessment of both QS signals has clearly indicated that anti-QS activity of the tested drugs is concentration dependent. Regarding the autoinducers $3 \mathrm{OH}-\mathrm{C} 12-\mathrm{HSL}$ and C4-HSL, they significantly decreased $(\mathrm{P}<0.01)$ by sub-inhibitory concentrations of cefepime, ceftazidime and imipenem compared to the untreated PAO1 strain (Figs. 1 and 2). In line with these findings, certain macrolide antibiotics (azithromycin) are capable of repressing the synthesis of $P$. aeruginosa AHLs signal molecules when applied at sub-MICs ${ }^{23}$. Furthermore, sub-inhibitory concentrations of tobramycin, ciprofloxacin, and ceftazidime were effective in lowering the AHL levels in P. aeruginosa ${ }^{24}$.The QSI of some antimicrobials is attributed to the alteration in membrane permeability, and subsequent elimination of the flux of N-3-oxo-dodecanoyl-1-homoserine lactone ${ }^{25}$. Also, efflux inhibitor phenylalanine arginyl $\beta$-naphthylamide caused significant elimination of QS signals and related virulence factors among multidrug resistant $P$. aeruginosa clinical isolates ${ }^{26}$.

To confirm that the elimination of QS signals and virulence was not related to the antimicrobial activities of the tested $\beta$-lactams, viable counts of the treated cells were performed and our data have shown no change in the cell growth. Bacterial growth in the presence of Sub-MICs of tested $\beta$-lactams produced viable bacterial count similar to the positive control (untreated $P$. aeruginosa PAO1).

The effect of sub-MIC concentrations on $P$. aeruginosavirulence has been verified. One of the main pathogenic factors in P. aeruginosa is LasB elastase. LasB elastase and proteases interfere with host defense mechanisms and destroy tissue components ${ }^{27}$. Most of sub-MICs of the tested compounds resulted in a significant decrease in LasB elastase and proteases enzymes $(\mathrm{P}<0.01$ and $\mathrm{P}<0.05)$ (Figs. 3-4). The inability of PAO1 strain to produce elastase and protease has been linked with their reduced level of associated QS molecules released under the influence of the assayed antibiotics. Much lower concentration (1/20 MIC) of some of these antibiotics did not affect bacterial QS signaling network and hence, showed no remarkable effect on elastase and protease activities. QS-deficient strains were non-virulent and lose the ability to produce elastase and protease ${ }^{28}$.

Hemolysin is another aspect of $P$. aeruginosa virulence which enhances its pathogenicity and helps in the sur- vival of pathogen by inhibiting the host defense factors. Significantly reduced levels $(\mathrm{P}<0.01)$ of hemolysin were observed after treating $P$. aeruginosa $\mathrm{PAO} 1$ with sub-inhibitory concentrations of cefepime, ceftazidime, and imipenem (Fig. 5). Similarly, a low hemolytic activity of $P$. aeruginosa was reported with sub-inhibitory concentrations of erythromycin ${ }^{29}$, eugenol ${ }^{30}$ and the 7 -fluoroindole ${ }^{17}$.

On the same instance, QS system coordinates the release of secondary metabolites such as pyocyanin ${ }^{15}$. Direct antagonists of QS in P. aeruginosa have a great impact on the production of pyocyanin ${ }^{31}$. In this study, the level of pyocyanin was significantly reduced $(\mathrm{P}<0.01)$ with sub-MICs of cefepime, ceftazidime and imipenem (Fig. 6).

These results indicated that controlling the production of QS molecules hinders most of the associated virulence agents. Also, inhibition of AHL signaling in Pseudomonas caused a reduction in the related virulence factors and attenuation of this pathogen when tested on animal models of pneumonia. On the same instance, synergistic effect has been achieved upon treating mice with both the QSI furanone C-30 and tobramycin, with increase in the clearance of $P$. aeruginosa in a foreign-body infection model $^{32}$.

\section{Conclusion}

The fact that these antibiotics are already approved drugs for human use is a significant benefit in the future application and development of anti-pathogenic drugs. Hence, further investigations are necessary to detect anti-quorum sensing potential of antimicrobial combinations. Also, further in-vivo studies are required to confirm their possible utilization as anti-pathogenic agents for competing with $P$. aeruginosa infections.

\section{Acknowledgements}

All appreciation to Prof. Martin Schuster, Department of Microbiology, Oregon State University, Nash Hall, Corvallis, OR 97331 for the E. coli reporter strain MG4/ pKDT17 and P. aeruginosa PAO-JP2. All thanks to Prof. Paul Williams, Department of Molecular Medical Science, Centre for Bimolecular Science, University of Nottingham, UK, for $P$. aeruginosa/ pME3846.

\section{Conflict of interest}

The authors have no conflict of interest to declare. 


\section{References}

1. Wagner VE, Filiatrault MJ, Picardo KF, Iglewski, BH. Pseudomonas aeruginosa virulence and pathogenesis issues. in: P Cornelis (Ed.) Pseudomonas genomics and molecular biology. 1st edn. Caister Academic Press, Norfolk; 2008: 129-158.

2. Pessi G, Williams F, Hindle Z, Heurlier K, Holden MT, Camara $\mathrm{M}$, et al. The global post transcriptional regulator RsmA modulates production of virulence determinants and $\mathrm{N}$-acylhomoserine lactones in P. aeruginosa. J Bacteriol 2001; 183: 6676-6683.

3. Pearson JP, Gray KM, Passador L, Tucker KD, Eberhard A, Iglewski BH, et al. Structure of the autoinducer required for expression of $\mathrm{P}$. aeruginosa virulence genes. Proc Natl AcadSci USA 1994; 91(1): 197-201.

4. Fuqua WC, Winans SC, Greenberg, EP. Quorum sensing in bacteria: the LuxR-LuxI family of cell density-responsive transcriptional regulators. J Bacteriol 1994; 176: 269-275.

5. Adonizio A, Kong KF, Mathee K. Inhibition of quorum sensing-controlled virulence factor production in P. aeruginosa by South Florida plant extracts. Antimicrob Agents Chemother 2008; 52: 198-203.

6. Zaki AA, Shaaban MI, Hashish NE, Amer MA, Lahloub MF. Assessment of Anti-Quorum Sensing Activity for Some Ornamental and Medicinal Plants Native to Egypt. Sci Pharm 2013; 81: 251-258.

7. Al-Haidari RA, Shaaban MI, Ibrahim S, Mohamed G. Anti-quorum sensing activity of some medicinal plants. Afr J Tradit Complement Altern Med 2016; 13(5):67-71.

8. Hassan R, Shaaban MI, Abdel Bar FM, El-Mahdy AM, Shokralla S. Quorum Sensing Inhibiting Activity of Streptomyces coelicoflavus Isolated from Soil. Front Microbiol 2016; 7: 659.

9. Gabr MT, El-Gohary NS, El-Bendary ER, El-Kerdawy MM, Ni N, Shaaban MI. Synthesis, antimicrobial, antiquorum-sensing and cytotoxic activities of new series of benzothiazole derivatives. CCL, 2015; 26: 1522-1528.

10. Nalca Y, Jänsch L, Bredenbruch F, Geffers R, Buer J, Häussler S. Quorum-sensing antagonistic activities of azithromycin in Pseudomonas aeruginosa PAO1: a global approach. Antimicrob Agents Chemother 2006; 50: 16801688.

11. Pearson JP, Pesci EC, Iglewski BH. Roles of Pseudomonas aeruginosa las and rhl quorum-sensing systems in the control of elastase and rhamnolipid biosynthesis genes. J Bacteriol 1997; 179: 5756-5767.

12. CLSI Clinical and Laboratory Standards Institute. Performance standards for antimicrobial susceptibility testing: 24 informational supplement M100-S24, CLSI, Wayne: PA 2014.

13. Standards Australia. Water Microbiology-Heterotrophic Colony Count Methods - Pour Plate Method using Plate Count Agar. AS 4276.3.1. Committee FT/20, Water Microbiology. Homebush, Australia: Council of Standards Australia, 1995. PubMed

14. El-Mowafy SA, Abd El- Galil KH, El-Messery SM, Shaaban MI. Aspirin isan efficient inhibitor of quorum sensing, virulence and toxins in Pseudomonas aeruginosa. Microb Pathog 2014; 74: 25-32.

15. Gupta RK, Setia S, Harjai, K. Expression of Quorum Sensing and Virulence Factors Are Interlinked in P. aeruginosa: an in vitro Approach. Am J Biomed Sci 2011; 3 (2): 116-125. PubMed

16. Miller JA. Experiments in molecular genetics. Cold Spring Harbor Laboratory Press, Plainview, NY, 1972.

17. Ohman DE, Cryz SJ, Iglewski. Isolation and characterization of a P. aeruginosa PAO1 mutant that produces altered elastase. J Bacteriol 1980; 142: 836-884 PubMed . 18. El-Mowafy SA, Shaaban MI, Abd El- Galil KH. Sodium Ascorbate as a Quorum Sensing Inhibitor of Pseudomonas aeruginosa. J Appl Microbiol 2014;117(5):13881399.

19. Rossignol G, Merieau A, Guerillon J, Veron W, Lesouhaitier O, Feuilloley MG, et al. Involvement of a phospholipase $C$ in the hemolytic activity of a clinical strain of P. fluorescens. BMC Microbiology 2008; 8:189.

20. Ra'oof WM, Latif IA. In vitro study of the swarming phenomena and antimicrobial activity of pyocyanin produced by P. aeruginosa isolated from different human infections. Europ J of scientific research 2010; 47 (3): 405.

21. Oberhardt MA, Puchalka J, Fryer KE, Martins dos Santos VA, Papin JA. Genome-Scale Metabolic Network Analysis of the Opportunistic Pathogen Pseudomonas aeruginosa PAO1. J Bacteriol 2008; 190: 2790-2803 PubMed .

22. Jimenez PN, Koch G, Thompson JA, Xavier KB, Cool RH, Quax, WJ. The Multiple Signaling Systems Regulating Virulence in Pseudomonas aeruginosa. Microbiol Mol Biol Rev 2012; 76 (1): 46-65 
23. Pechere JC. Azithromycin reduces the production of virulence factors in Pseudomonas aeruginosa by inhibiting quorum sensing. Jpn J Antibiot 2001; 54:87-89.

24. Garske LA, Beatson SA, Leech AJ, Walsh SL, Bell SC. Sub-inhibitory concentrations of ceftazidime and tobramycin reduce the quorum sensing signals of $P$. aeruginosa. Pathology 2004; 36 (6): 571-575.

25. Skindersoe ME, Alhede M, Phipps R, Yang L, Jensen PO, Rasmussen TB, et al. Effects of Antibiotics on Quorum Sensing in P. aeruginosa. Antimicrob Agents Chemother 2008; 52: 3648-3663.

26. El-Shaer S, Shaaban M, Barwa R, Hassan R. Control of quorum sensing and virulence factors of Pseudomonas aeruginosa using phenylalanine arginyl -naphthylamide. J Med Microbiol. 2016 in press.

27. Khajanchi BK, Sha J, Kozlova EV, Erova TE, Suarez G, Sierra JC, et al. N-acylhomoserine lactones involved in quorum sensing control the type VI secretion system, biofilm formation, protease production, and in vivo virulence in a clinical isolate of Aeromonas hydrophila. Microbiology 2009; 155: 3518-3531 PubMed .

28. Wilder CN, Allada G, Schuster, M. Instantaneous within-patient diversity of P. aeruginosa quorum-sensing populations from cystic fibrosis lung infections. Infect Immun 2009; 77(12): 5631-5639. PubMed

29. Sofer D, Gilboa-Garber N, Belz A, Garber NC. Subinhibitory' erythromycin represses production of Pseudomonas aeruginosa lectins, autoinducer and virulence factors. Chemotherapy 1999; 45: 335-341.

30. Harjai K, Kumar R, Singh S. Garlic blocks quorum sensing and attenuates the virulence of Pseudomonas aeruginosa. FEMS Immunology Medical Microbiology 2010; 58: 161-168.

31. Morkunas B, Galloway WR, Wright M, Ibbeson BM, Hodgkinson JT, O'Connell KM, et al. Inhibition of the production of the P. aeruginosa virulence factor pyocyanin in wild-type cells by quorum sensing autoinducer-mimics. Organic Biomol Chem 2012; 42: 8452-8464.

32. Christensen LD, van Gennip M, Jakobsen TH, Alhede M, Hougen HP, Høiby N, et al. Synergistic antibacterial efficacy of early combination treatment with tobramycin and quorum-sensing inhibitors against Pseudomonas aeruginosa in an intraperitoneal foreign-body infection mouse model. J Antimicrob Chemother 2012; 67: 1198-1206. 